\title{
Frustration-Driven Quantum Phase Transition in the 1D Extended Anisotropic Heisenberg Model
}

\author{
E. Plekhanov, A. Avella and F. Mancini \\ Dipartimento di Fisica "E.R. Caianiello" — Unità CNISM di Salerno \\ Università degli Studi di Salerno, 84081 Baronissi (SA), Italy \\ By using density matrix renormalization group technique we study the \\ 1D extended anisotropic Heisenberg model. We find that starting from the \\ ferromagnetic phase, the system undergoes two quantum phase transitions \\ induced by frustration. By increasing the next-nearest-neighbor interaction, \\ the ground state of the system changes smoothly from a completely polarized \\ state to a next-nearest-neighbor correlated one. On the contrary, letting the \\ in-plane interaction to be greater than the out-of-plane one, the ground state \\ changes abruptly.
}

PACS numbers: 75.10.Jm, 75.30.Kz, 75.10.Pq, 75.40.Mg

\section{Introduction}

Quantum phase transitions (QPTs), contrarily to ordinary ones (at $T \neq 0$ ), are characterized by a change of ground state on continuous varying the external conditions [1]. Such phase transitions are often caused by frustration. Onedimensional quantum spin systems offer a wide variety of examples of QPT. Among them, the Heisenberg-like models are the most important. While 1D Heisenberg model with only nearest-neighbor interaction possesses a well studied analytic solution by Bethe anzatz [2], the addition of next-nearest-neighbor (NNN) term suffices to break the integrability. In such extended system a whole set of new phenomena occurs. The physics of this model has been already largely studied by means of many field-theoretical as well as numerical methods [3], primarily in the proximity of the antiferromagnetic phase.

However, the ferromagnet is also one of the ground states of the model and it is primarily promoted by the diagonal part of the Hamiltonian. If other terms in the Hamiltonian induce frustration, then eventually the ferromagnet will "melt", and the system will undergo a QPT towards some new phase. In the present work we investigate such melting driven by two distinct frustrating factors: (i) the anisotropy of the nearest-neighbors interaction term and (ii) the antiferromagnetic NNN interaction. 


\section{Model and method}

We study the one-dimensional anisotropic extended Heisenberg model with NNN interaction

$$
H=-J_{z} \sum_{i} S_{i}^{z} S_{i+1}^{z}+J_{\perp} \sum_{i}\left(S_{i}^{x} S_{i+1}^{x}+S_{i}^{y} S_{i+1}^{y}\right)+J^{\prime} \sum_{i} \boldsymbol{S}_{i} \boldsymbol{S}_{i+2} .
$$

We find the ground state of the Hamiltonian (1) numerically by means of the density matrix renormalization group (DMRG) [4] technique on a chain with 100 sites, subject to open boundary conditions. We use $J_{z}>0$, which corresponds to ferromagnetic coupling. In the present work we calculate the Fourier transform of the correlation functions, both in-plane $\left(\left\langle S^{x}(k) S^{x}(k+q)\right\rangle\right)$ and out-of-plane $\left(\left\langle S^{z}(k) S^{z}(k+q)\right\rangle\right)$. Due to the large system size, the above correlation functions do not depend on $k$, but only on the transferred momentum $q$. The starting point for our analysis is the doubly degenerate completely polarized state with all spins either "up" or "down", located on the phase diagram of the system within the limits $0<J_{\perp}<J_{z}$ and $J^{\prime} \lesssim 0.31 J_{z}$, as found in Ref. [5].

\section{Frustration due to $J^{\prime}$}

Up to the values of $J^{\prime} \approx 0.31 J_{z}$ the system remains totally polarized. In the momentum space this corresponds to a $\delta$-function peak at $q=0$ in $z$-channel and a constant value of $1 / 4$ in the perpendicular one. On the other hand, in the limit $J^{\prime} \rightarrow \infty$ the system behaves as a couple of non-interacting antiferromagnetic Heisenberg chains with nearest-neighbor interaction. It is clear that in the case of $J^{\prime} \rightarrow \infty$ the system will exhibit short-range AF correlations with exponential decay in real space, which corresponds, in momentum space to a couple of Lorentzian peaks centered at $q= \pm \pi / 2$.

Smooth change of $J^{\prime}$ causes a smooth rearrangement of the ground state. Namely, for $J^{\prime} \gtrsim 0.31 J_{z}$ the system abandons the totally polarized state, although conserving much of the magnetization along the $z$-axis (see the peaks at $q=0$ in Fig. 1a for $J^{\prime}>0.31 J_{z}$ ). At the same time a short-ranged incommensurate phase appears: two strong Lorentzian-type peaks develop in the vicinity of $q= \pm \pi / 2$, the larger is $J^{\prime}$ the closer are the peaks to $\pm \pi / 2$.

On the contrary, in the perpendicular channel we have perfect commensurability (at least for the values of $J_{\perp} \lesssim 0.5 J_{z}$ ) as shown in Fig. 1b. As in the case of the $z$-axis, the in-plane correlations appear to decay exponentially with the correlation length depending on both $J_{\perp}$ and $J^{\prime}$.

\section{Frustration due to $J_{\perp}$}

Unlike to the smooth change of the properties in the previous case, frustration caused by $J_{\perp}$ changes the phase abruptly. The spins, totally polarized along $z$ direction for $J_{\perp}<J_{z}$, become antiferromagnetically ordered in the $x-y$ plane (see Fig. 1c). The remaining $z$-component of the spins remains uncorrelated 



Fig. 1. Momentum space spin-spin correlation functions for two representative lines in $J^{\prime}-J_{\perp}$ plane both along $z$ direction and in-plane: part (a) and (b) $J_{\perp}=0.2 J_{z}$ and $J^{\prime} \in[0.27,0.53]$; part (c) and (d) $J^{\prime}=0.2 J_{z}$ and $J_{\perp} \in[1,1.2]$.

already at few lattice spacings. In the limit $J_{\perp} \rightarrow \infty$ we expect the system to become the $X X$ model, with an in-plane $\mathrm{AF}$ ground state. As a matter of fact, at $J_{\perp}=1.2 J_{z}$ most of the correlations are concentrated in the $x-y$ plane. The scaling behaviour in the $x-y$ plane is once again exponential (Lorentzian in $k$ space, see Fig. 1d). The value of $J^{\prime}$ does not qualitatively change this picture within the range considered $\left(0<J^{\prime}<0.3 J_{z}\right)$. Beyond this range the two phases described above, should merge at some transition line, situated somewhere in the region $J^{\prime}>0.31 J_{z}, 0.5 J_{z}<0.9 J_{\perp}<J_{z}$.

\section{Summary}

In conclusion, by using a high-precision numeric tool (DMRG) we have demonstrated how different types of frustration can lead to QPTs in the 1D extended anisotropic Heisenberg model. By considering a chain with 100 sites we have been able to observe the exponential decay of the correlations and obtain a high resolution picture of the static spin-spin correlations in momentum space. In contrast to the frustration induced by $J_{\perp}$, the one due to $J^{\prime}$ leads to a rather smooth rearrangement of the ground state. A more detailed study of the 
two phases described in the present work and the transition between them is in progress and will be reported elsewhere.

\section{References}

[1] S.L. Sondhi, S.M. Girvin, J.P. Carini, D. Shahar, Rev. Mod. Phys. 69, 315 (1997).

[2] H. Bethe, Z. Phys. B 71, 205 (1931); L. Hulten, Arkiv Mat. Astron. Fyz. B 25A, 1 (1938).

[3] See e.g. F.D.M. Haldane, Phys. Rev. B 25, 4925 (1982); L. Capriotti, F. Becca, S. Sorella, A. Parola, Phys. Rev. B 67, 172404 (2003); A. Chubukov, Phys. Rev. B 44, 4693 (1991).

[4] S.R. White, Phys. Rev. Lett. 69, 2863 (1992); Phys. Rev. B 48, 10345 (1993).

[5] E. Plekhanov, A. Avella, F. Mancini, Phys. Rev. B 74, 115120 (2006). 